\title{
Erratum to: Effects of elicitors on trichothecene accumulation and Tri genes expression in potato tubers inoculated with Fusarium sulphureum
}

\author{
Xue Hua-Li • Bi Yang • Zong Yuan-Yuan • \\ Alejandro Calderón-Urrea • Wang Hu-Jun • \\ Pu Lu-Mei • Wang Yi • Li Yong-Cai
}

Published online: 12 May 2017

(C) Koninklijke Nederlandse Planteziektenkundige Vereniging 2017

\section{Erratum to: Eur J Plant Pathol}

DOI: $10.1007 / \mathbf{s} 10658-016-1125-1$

"This work was funded by the Ministry of Science and Technology of China (2016YFD04009-05)" to "This work was funded by the National Key Research and Development Program of China (2016YFD04009)". Actually, the relationship between them is that the project (No.2016YFD04009-05) is sub-project of the project (No.2016YFD04009), The authors declare no competing financial interests.

The online version of the original article can be found at http://dx. doi.org/10.1007/s10658-016-1125-1

X. Hua-Li • W. Hu-Jun • P. Lu-Mei

College of Science, Gansu Agricultural University,

Lanzhou 730070, People's Republic of China

B. Yang $(\bowtie) \cdot Z$. Yuan-Yuan $\cdot$ W. Yi $\cdot$ L. Yong-Cai

College of Food Science and Engineering, Gansu Agricultural

University, No.1 Yingmen road, Anning Distract,

Lanzhou 730070, People's Republic of China

e-mail: biyang@gsau.edu.cn

X. Hua-Li

College of Plant Protection, Gansu Agricultural University,

Lanzhou 730070, People's Republic of China

A. Calderón-Urrea

Department of Biology, California State University, Fresno, CA

93711, USA 